\title{
Multi-directionally wrinkle-able textile OLEDs for clothing-type displays
}

Seungyeop Choi', Woosung Jo², Yongmin Jeon', Seonil Kwon', Jeong Hyun Kwon'1, Young Hyun Son', Junmo Kim², Jun Hong Park', Hyuncheol Kim', Ho Seung Lee', Minwoo Nam¹, Eun Gyo Jeong' ${ }^{1}$, Jeong Bin Shin', Taek-Soo Kim² and Kyung Cheol Choi $\mathbb{1}^{1 凶}$

A clothing-type wearable display can be utilized in fashion, bio-healthcare, and safety industries as well as smart textiles for the internet of things (IoTs) and wearable devices. In response to this trend, we demonstrate a textile display that can endure the active movements of a human body. It can be applied to any kind of textile, and is durable against conditions such as rain, sweat, and washing. As a key technology for realizing the multi-directional wrinkle-able textile display, we fabricated a stress-lowering textile platform with an ultrathin planarization layer replicated from the flat surface of glass. An elastomeric strain buffer for reducing mechanical stress is also inserted into the textile platform. Here, organic light-emitting diodes (OLEDs) with red, green and blue color, thin film transistors (TFTs) fabricated at a low temperature below $150^{\circ} \mathrm{C}$, and a washable encapsulation layer blocking both gas and liquid were demonstrated on the textile platform.

npj Flexible Electronics (2020)4:33; https://doi.org/10.1038/s41528-020-00096-3

\section{INTRODUCTION}

Wearable displays are likely to become a core part of mobile, wearable and internet of things (loTs) devices, becoming a key technology of the fourth industrial revolution in the 21 st century. The information display is the most efficient and intuitive communication device between a human and an electronic device, thus it is very useful when it is applied into a wearable device. Wearable displays have been developed in the form of watches, bands, bracelets, and are now evolving into a textile form with softness and comfortable fit. It is a specialty of textiles to be able to deform freely depending on the curvature and movement of a human body. A film-type substrate is only bendable unidirectionally, but lowering its thickness to enhance flexibility seriously reduces its durability. However, textiles have the advantages of both high flexibility and durability based on a woven structure. Everyone wears clothes made of textiles every day, and this proves that textiles are the ideal wearable display platforms for humans. The textile-based wearable display can have both functionality and esthetic value, and it can be incorporated into many practical applications, such as smart fashion, a stage costume, a dress, curtains, tablecloths, and automobile inner walls. Recently, a number of information and communication technology (ICT) devices have been converted to be more human-centered technologies. Continuing this trend, textile displays can be a growth engine for the global wearable market.

Clothing-type displays can be fabricated in two ways, from a single fiber ${ }^{1}$ and from a textile ${ }^{2}$. Cylindrical fiber-shaped-emitting devices $^{3-10}$ have strengths in terms of breathability and texture when they are woven into a textile. However, clear solutions have not been suggested yet to address their circuit configuration, weaving method, and the active matrix (AM) operation. Textilebased displays, on the other hand, have strong points in terms of feasibility and practicality ${ }^{11,12}$. The first clothing-type textile displays based on light-emitting diodes (LEDs) attached to the textile were released several years ago ${ }^{13}$. However, LEDs are too large for high-resolution displays, and their rigidity can cause serious discomfort. For a better wearing sensation, thin film-type light sources with lightweight and flexibility have been reported. Initially, the textile was flattened by repetitive inkjet printing, and the fabricated AC powder electroluminescent (EL) device showed a luminance of $40 \mathrm{~cd} \mathrm{~m}^{-2}$ at $440 \mathrm{~V}$ with a thickness of about $200 \mu \mathrm{m}^{14}$. Then, $30-70 \mu \mathrm{m}$-thick polyurethane (PU) and polyethylene naphthalate (PEN) films were thermally laminated to the textile, and flexible organic light-emitting diodes (OLEDs) were fabricated on the planarized textile ${ }^{15-18}$. In addition, a screenprinted 7-segments watch display using about $150 \mu$ m-thick planarization and EL devices ${ }^{19}$ and an OLED integrated with a polymer solar cell (PSC) on polyethylene terephthalate (PET) filmattached textile for self-powered, wearable displays ${ }^{20,21}$ have been reported. The previous works using the inkjet printing, the screen printing, thermal lamination, etc., required thick planarization layers with a micrometer-scale thickness to flatten the bumpy textile, and this caused the textile to have plastic-like mechanical behavior.

Here, we report the fundamental technologies of a textile-based OLED display which has wide applicability, washability, as well as mechanical flexibility like real textiles. In this work, the textile platform had a simple structure, with an ultrathin planarization layer, a strain buffer, and a polyester textile. Nonetheless, by using a differentiated fabrication process and a stress-lowering structure, it showed greatly improved mechanical properties and practicality. By employing a surface-replicating method with a sacrificial layer, a glass-like flat surface could be formed on the textile with only a $0.5 \mu \mathrm{m}$-thick planarization layer. In addition, a strain buffer was introduced to reduce the strain transferred from the textile to the planarization layer, and as a result the textile platform showed mechanical reliability under wrinkling as well as nearly folding. Red, green, and blue colored OLEDs, a lowtemperature designed thin film transistor (TFT) and a waterresistant encapsulation were demonstrated on the textile platform.

${ }^{1}$ School of Electrical Engineering, Korea Advanced Institute of Science and Technology (KAIST), Daejeon 34141, Republic of Korea. ${ }^{2}$ Department of Mechanical Engineering, Korea

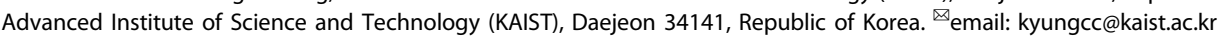


a
1. Flat substrate (glass)

\section{Sacrificial layer (PVA)} - spin coating \& bake

3. Ultrathin planarization layer (SU-8) - spin coating \& soft bake, exposure, PEB

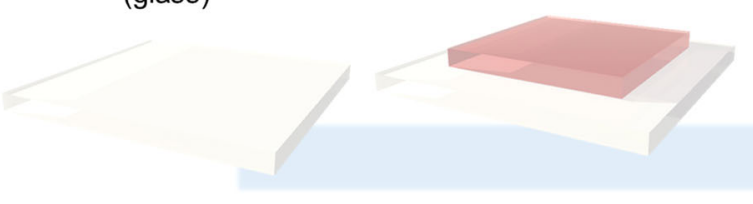

"Stress-lowering structured
flexible textile platform" und tom

7. Natural drying
6. Removing sacrificial layer by deionized water
4. Strain buffer

- spin coating b
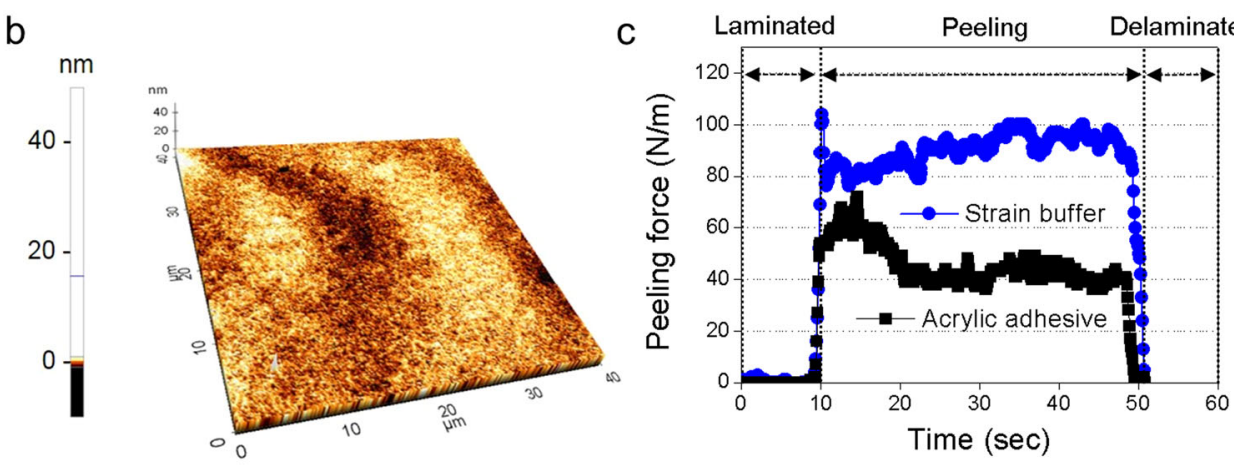

e

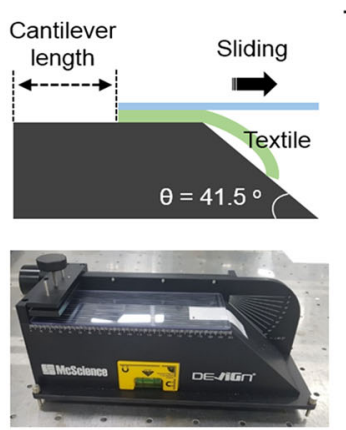

f

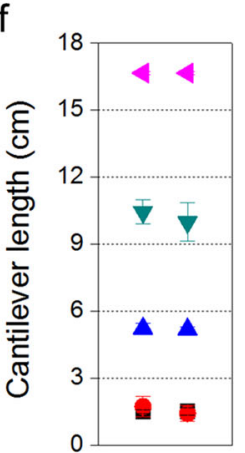

9

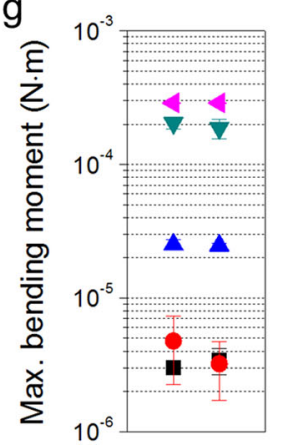

h

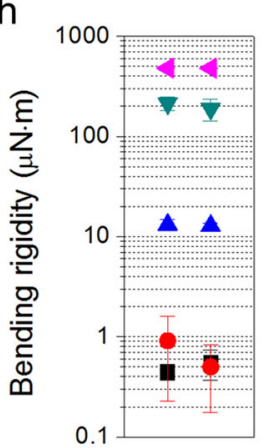

Uniform

pressure $\downarrow \downarrow \downarrow$

of $10 \mathrm{~N}$

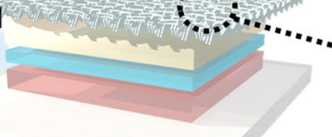

5. Lamination of textile

\& Natural curing of strain buffer

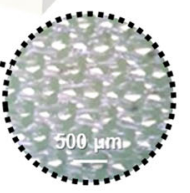

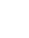


layers were minimized to enhance the flexibility of the textile platform.

This process is very simple but it has powerful merits. First, there is no need for a thick planarization which reduces flexibility. In previous works, the thicknesses of the inkjet-printed, the screenprinted and thermally laminated planarization layer was about 200,150 , and $30-70 \mu \mathrm{m}$, respectively. On the other hand, in this study, the $230 \mu \mathrm{m}$-thick textile was perfectly flattened by the $0.5 \mu \mathrm{m}$-thick planarization layer by using the proposed surfacereplicating process. An atomic force microscopy (AFM) image of the surface of the textile platform is shown in Fig. 1b. $R_{\text {rms }}$ (rootmean-square surface roughness) and $R_{\mathrm{p}-\mathrm{v}}$ (peak-to-valley surface roughness) values were measured to be 0.45 and $29.26 \mathrm{~nm}$, respectively. In other words, a plane surface like a conventional glass substrate was perfectly realized on the textile through the only $0.5 \mu \mathrm{m}$-thick planarization layer. Second, the strain buffer basically functions as an adhesive. To evaluate the adhesion ability of the strain buffer, a peel test was conducted (Supplementary Fig. 1). In this test, a force to delaminate the textile from a substrate, a peeling force, was measured. As seen in Fig. 1c, the strain buffer showed a stronger adhesion ability than a generally used acrylic adhesive having chemical bonding. The average peeling forces by the acrylic adhesive and the strain buffer were 45.4 and $88.9 \mathrm{~N} / \mathrm{m}$, respectively. The strain buffer as a Si-base elastomer has properties similar to those of general rubber, with scant chemical adhesive force. Therefore, a means by which to generate physical adhesion between the strain buffer and the textile was designed. First, the strain buffer in a liquid state was coated onto the planarization layer, and it hardened after sufficiently permeating into the textile. This process generated interlocking of the strain buffer within the textile. This mechanism induced physical bonding and enhanced the adhesion between the strain buffer and the textile. In addition, plastic deformation of the strain buffer consumes more energy, thus the larger peeling force was required. At the same time, the strain buffer also greatly reduces the strain transfer from the textile to the planarization layer. This enables wrinkling and modest stretching of the textile platform (Supplementary Fig. 2). Third, the proposed fabrication method provides benefits with respect to cost and process time. The textile platform was fabricated by only solution process. It has advantages in mass production, and low cost methods, such as bar coating, reel-to-reel, etc., can be employed here. Last, the textile platform had a multi-stack structure, but no internal stress was observed. Large internal stress can cause the material to be damaged by small external force, thus the design for zero internal stress is important. As shown in Fig. 1d, the internal tensile stress, $26.39 \mathrm{MPa}$, was generated by hardening process of the planarization layer, SU-8. It was released to $4.65 \mathrm{MPa}$ by the strain buffer. After the thick textile with the zero-stress was laminated, and the internal stress of the SU- 8 was shared to the whole platform and became nearly zero, $0.08 \mathrm{MPa}$ (Supplementary Fig. 3).

The flexibility of the textile platform was evaluated using the cantilever test (ASTM:D1388) ${ }^{22}$. As shown in Fig. 1e, when the textile sample is slid in the direction of the incline, it is bent downward by gravity and finally touches the slope. The length of the bent part is defined as the cantilever length. A more flexible textile has a shorter cantilever length. In this test, all textiles are assumed to be a plate because the cantilever test was developed based on a beam and plate theory. The cantilever lengths of the bare polyester textile, the textile platform, PET films, and an A4 paper are shown in Fig. 1f. In the case of the textile platform, bending up and down have different values, because the planarization layer and the strain buffer are formed on top of the textile. Therefore, cantilever lengths for both up and down were measured. The notable thing is that the bare textile and the textile platform had almost the same cantilever lengths. However, since the cantilever length is affected by the weight of the sample, the cantilever length is not completely consistent with the sample's flexibility. As shown in Fig. 1g, the maximum bending moments affecting the cantilever lengths were all different because all of the samples had different weights. Hence, in the cantilever test, the textile was modeled as a plate, and the mechanical behavior of the plate was calculated based on plate theory of applied mechanics. The bending rigidity, which means the actual mechanical flexibility, was calculated here (Supplementary Equations) $)^{23-25}$. Through this method, it was confirmed that the textile platform had almost the same mechanical flexibility as the bare textile, as shown in Fig. 1h. Furthermore, each Young's modulus was calculated using the bending rigidity and the thickness. The Young's moduli of the bare textile were 0.52 and $0.64 \mathrm{MPa}$, and that of the textile platform were 0.50 and $0.28 \mathrm{MPa}$. The planarization layer with a thickness of $0.5 \mu \mathrm{m}$ hardly affected the mechanical flexibility of the whole textile platform, and the strain buffer also did not change the mechanical properties of the textile because the strain buffer and the textile had the same Young's modulus value of about $0.3 \mathrm{MPa}$. The Young's modulus values were utilized to simulate the bending of the textile platform.

\section{Strain reduction effect by the strain buffer}

The textile platform was designed to transfer as little strain as possible to the planarization layer from the textile when the textile was bent, wrinkled, and stretched. The relationships between the strain and stress of the textile platform and each layer are shown in Fig. 2a and Supplementary Fig. 4. Young's modulus of the bare textile in the tensile test was almost the same as in the cantilever test, while that of the textile platform in the two tests was a little different. The effective Young's modulus of the textile platform by the cantilever test was $0.39 \mathrm{MPa}$ (average), and that by the tensile test was $6.71 \mathrm{MPa}$. It shows that the mechanical property of the textile platform was closer to the textile in terms of flexibility than stretchability. The most meaningful part of the tensile test was that the SU-8 in the textile platform started to crack at a strain of $3.88 \%$, although the single layer SU-8 was broken at a strain of $0.77 \%$ (Fig. $2 \mathrm{~b}-\mathrm{d}$ ). In other words, the strain buffer effectively reduced the strain generated by the textile, and transferred reduced strain by one-fifth to the planarization layer. The thicker strain buffer can enhance mechanical properties related to the stretchability, but it lowers the flexibility of the textile platform. In this study, flexible characteristics of the textile platform were more important in order to realize wrinkle-able textile displays. Thus, the optimum thickness of the strain buffer was the minimum thickness having the physical bonding between the textile and the planarization layer. When a strain of more than $6 \%$ was applied, it was observed that the planarization layer and the strain buffer delaminated from the textile due to the large shear strain.

\section{Bending and wrinkling analyses of the textile-OLED}

Mechanical deformations of the textile platform-based OLED (for brevity, textile-OLED) was evaluated by repetitive bending and wrinkling. The textile-OLED showed the same luminance-voltage and current-voltage characteristics as the glass-OLED, and could be operated stably up to a bending radius of $1.5 \mathrm{~mm}$ in compressive bending, and $2 \mathrm{~mm}$ in the tensile (Fig. 3a). After tensile bending at a radius of $1.5 \mathrm{~mm}$ the textile-OLED was still able to emit light but it had critical cracks, as seen in Fig. 3b. It was very meaningful that the $235-240 \mu$ m-thick textile-OLEDs showed reliable operation under bending radii of $2 \mathrm{~mm}$ or less. OLEDs on plastic films with the same thickness as the textile platform hardly work under bending radii of $<20 \mathrm{~mm}$ because OLEDs are difficult to operate under a strain of about $1 \%$.

The strain in each layer of the bent textile platform was analyzed by finite-element method (FEM)-based mechanical simulation (Fig. 3c). The textile was modeled as a plate in order to apply Young's modulus values obtained from the cantilever 
a

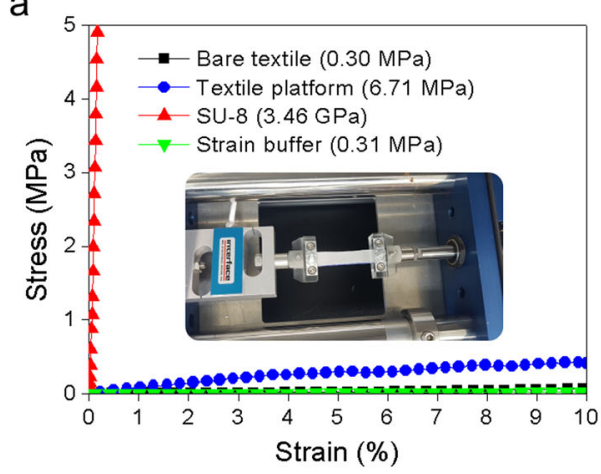

d

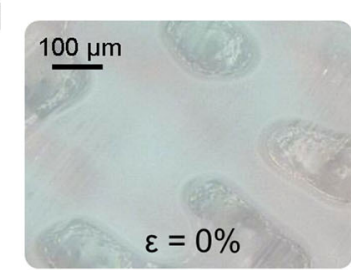

b

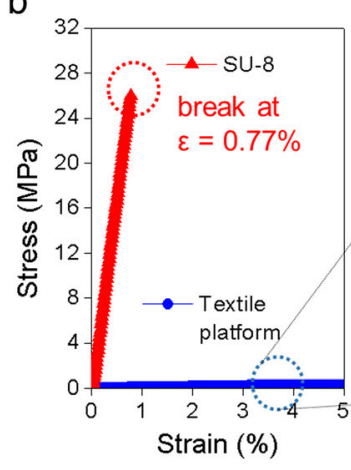

C

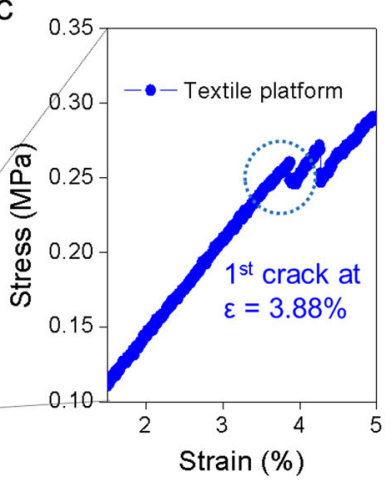

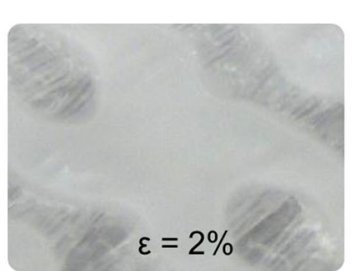
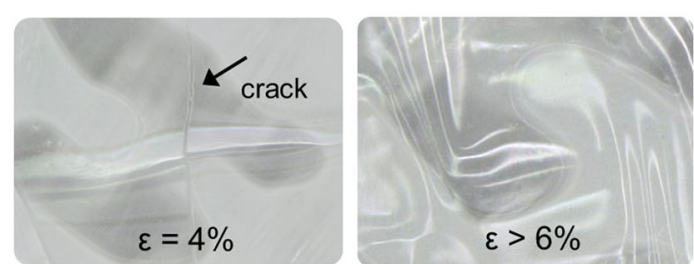

Fig. 2 Tensile tests of the textile platform. a The mechanical stress along the strain within the textile platform and each layer, calculated from the tensile test results. $\mathbf{b}$ Comparison of the mechanical stress between the single SU-8 layer and the textile platform. $\mathbf{c}$ Expanding the strain-stress graph of the textile platform. d Optical microscopy images of the textile platform after tensile testing at strains of $0 \%$, $2 \%$, $4 \%$, and more than $6 \%$.

test. The thickness of the textile was as many as about $230 \mu \mathrm{m}$, and bending strain is largely affected by thickness. Nevertheless, the strain buffer and the textile effectively contributed to reduce the strain on the planarization layer. In the simulational results, a maximum principal strain of about $4.4 \%$ occurred in the textile, but in the planarization layer it was reduced to about $1.3 \%$. This shows that the neutral axis of the textile platform is positioned near the surface of the planarization layer, because the strain buffer and the textile had very low Young's modulus values of $0.3 \mathrm{MPa}$.

In this work, we proposed a wrinkling test in which four displacement supports move on a plane. This was inspired by the bending test in which one displacement support moves in one axis (Supplementary Fig. 5). The fixed support on the backside of the textile-OLED helped the sample to wrinkle, not bend, and the four displacement supports moved to the center to create wrinkles. In the bending test, the bending radius, which indicates how harsh the applied strain is, is determined by the distance between the two supports. Similarly, the strength of the wrinkles was expressed as the relative area marked by the four displacement supports. As seen in Fig. 3d and e, harsh wrinkling resulted in a luminance reduction of about $15-20 \%$. The textileOLEDs still showed more than $800 \mathrm{~cd} \mathrm{~m}^{-2}$, the luminance required for outdoor displays, after multi-directional wrinkling. The wrinkles were repeated in the initial shape, and the decrease of the luminance saturated after about 50 repetitions. The distribution of strain in the wrinkled textile-OLED was visualized using the digital image correlation (DIC) technique (Supplementary Fig. 6). In DIC, randomly positioned dots are formed on the surface of the textile platform first, and each dot is mapped to trace the shape of the textile platform. The amount of strain was calculated by changes in the position of the dots before and after the wrinkling. As seen in Fig. $3 f-i$, wrinkling caused non-uniform strain distributions, and very high strains were generated at the local area. The maximum principal strains in the OLED cells were about $1.9 \%$ and $5.1 \%$ for the relative cell areas of $75.1 \%$ and $53.7 \%$, respectively. Tensile stress was generated on the outside, and compressive stress on the inside, near the center. Actually, OLED cannot be operated under the tensile strain more than $1 \%$. Most of organic materials and polymers can endure a larger compressive stain than a tensile strain, thus the wrinkled textile-OLED can emit the light under the strain more than $5 \%$.

\section{Practical applications of the textile-display}

First, red $(R)^{26}$, green $(G)$ and blue $(B)^{27-30}$ colored OLEDs were fabricated on the textile platform to enable it to produce colorful images and videos (Fig. 4a). The RGB colored textile-OLEDs operated stably under the harsh mechanical stress near the folding. The RGB textile-OLEDs fabricated at $69 \mathrm{~mm}^{2}$ also showed operational reliability. This means that the ultrathin SU-8 maintained a significantly flat surface over a large area. As shown in Fig. 4b, the glass-OLED and textile-OLEDs exhibited the same emission characteristics. If the thickness or the quality of the metals and the organics in the OLEDs on the glass and the textile platform had been different, it would have caused a difference in the current density, the luminance, and the micro-cavity effects (Supplementary Fig. 7).

Second, polymer light-emitting diodes (PLEDs) and TFTs were demonstrated on the textile platform. The textile-PLEDs have advantages of low cost and mass production, because all of the fabrication processes, from the textile platform to the PLEDs, can be accomplished by solution process. Wetting of the textile by solutions was inevitable, however, and it caused a little deformation of the textile. Thus, there was a slight difference in electrical and optical (JVL) characteristics, as shown in Fig. 4c. Nevertheless, the fabricated textile-PLED exhibited a high maximum luminance of $91,970 \mathrm{~cd} \mathrm{~m}^{-2}$. In Fig. $4 d$, a textile-TFT as a core device for an AM-OLED was demonstrated. Actually, heat-sensitive textiles are not compatible with TFTs which generally need a hightemperature annealing process. This was the reason why the SU-8 with a low CTE and high $T_{\mathrm{g}}$ was employed as the planarization layer of the textile platform. In addition, a TFT fabrication method on the textile platform was designed to lower the process temperature, and maintain good mobility and switching characteristics. Because a bottom-gate TFT needs a high-temperature annealing process to recover the damage produced by the sputtering process, a top-gate structure that is relatively less dependent on a heating process was applied as the textile-TFT. To further reduce the annealing dependency, an 


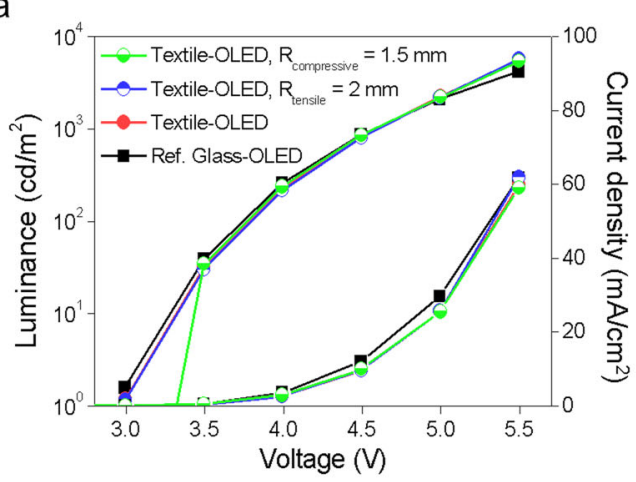

b
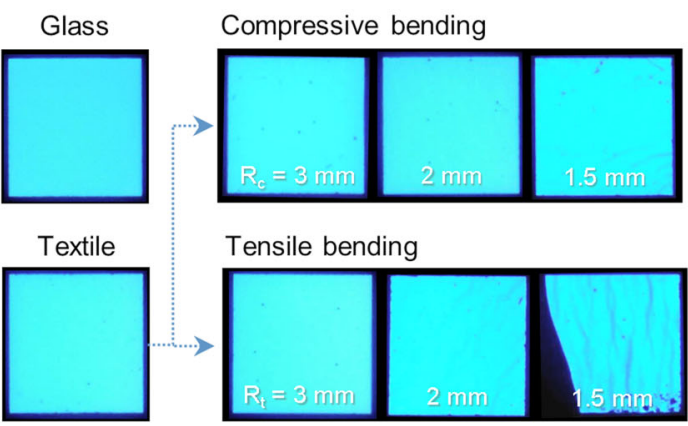

C

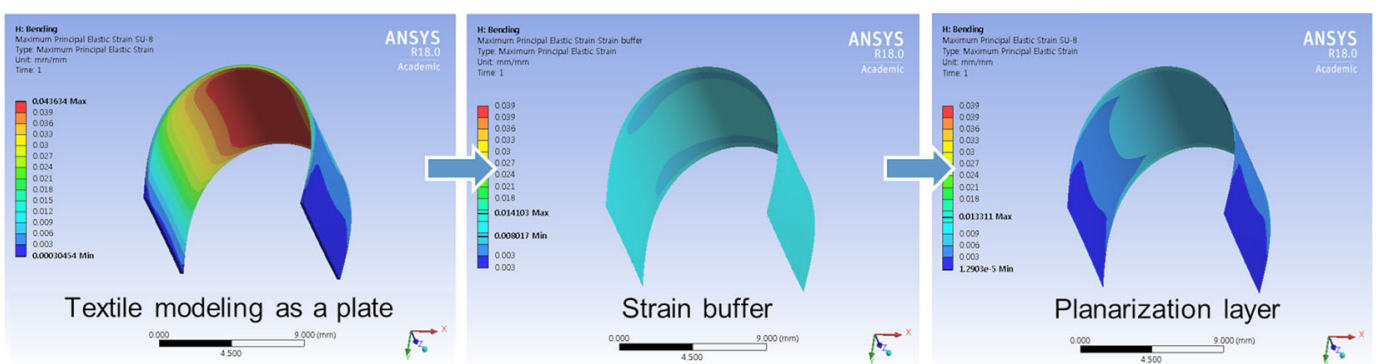

d

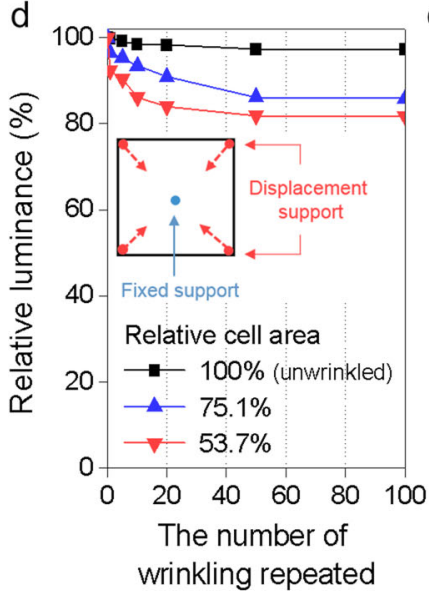

e

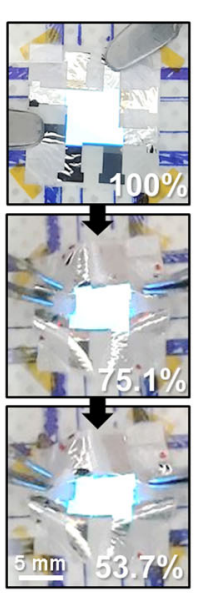

f

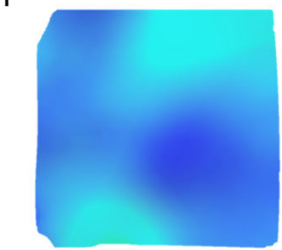

h

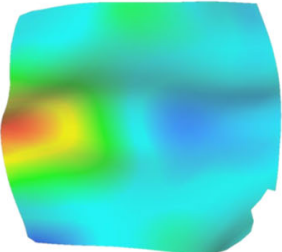

g

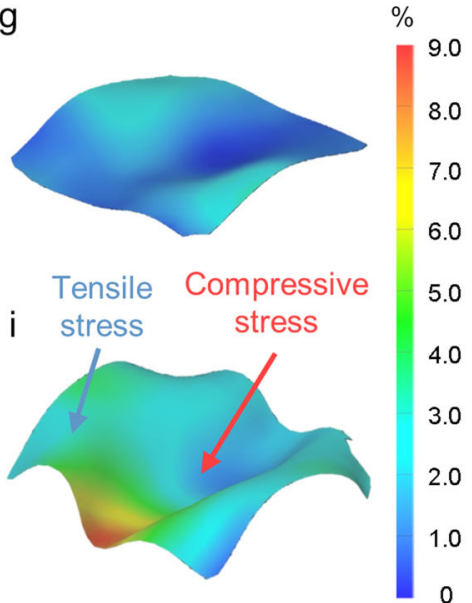

Fig. 3 Bending and wrinkling characteristics of the textile-OLEDs. a Repetitive bending test of the textile-OLEDs in the tensile and compressive directions, the number of bendings: 1000 (reference: OLED fabricated on the glass substrate). $\mathbf{b}$ The cell images of the textileOLEDs after bending. $\mathbf{c}$ Strain analyses of each layer within the bent textile platform using FEM mechanical simulation. $\mathbf{d}$ Repetitive wrinkling test of the textile-OLEDs, the initial luminance: 1000 nit (inset: schematic diagram of how the wrinkling test was conducted). e The cell images of the textile-OLEDs after wrinkling. $\mathbf{f}-\mathbf{i}$ Strain analyses of the wrinkled textile-OLEDs using DIC, the relative cell area of $75.1 \%$ in the top view and the arbitrary view, the relative cell area of $53.7 \%$ in the top view and the arbitrary view.

insulator was formed by atomic layer deposition (ALD) at $150^{\circ} \mathrm{C}$, because a thin film with high insulating properties can be formed by the ALD process at a relatively low temperature. In addition, each layer was patterned just by shadow mask in order to avoid photolithography in which highly reactant solvents are used. The use of harmful solvents is not suitable for fabrication process of clothing-type devices because users can wear it for a long time. The textile-TFT showed a mobility of $14.9 \mathrm{~cm}^{2} \mathrm{~V}^{-1} \mathrm{~s}^{-1}$ in the saturation mode (drain voltage $=10.1 \mathrm{~V}$ ), a subthreshold swing (SS) of $135.1 \mathrm{mV} /$ decade, an on-off ratio of $5.11 \times 10^{8}$ and $V_{\text {th }}=$ $1.66 \mathrm{~V}$.

Third, water-resistant and washable textile-OLEDs fabricated with a gas barrier and liquid passivation were demonstrated, as shown in Fig. 4e. The gas barrier and the OLED were deposited on the textile platform, and the liquid passivation on which the gas barrier had formed was laminated to the OLED. The gas barrier that functioned as the OLED encapsulation to block moisture and oxygen $^{31}$ was fabricated using a spacer polymer ${ }^{32,33}$ and the nano-stratified barrier of $\mathrm{Al}_{2} \mathrm{O}_{3}$ and $\mathrm{ZnO}$ quasi-films ${ }^{34-38}$. The porous and defect-decoupling characteristics of the nanostratified structure enhanced the structural flexibility and barrier property. This barrier was fabricated as 3.5 dyads with an alternative deposition of a polymeric solution, and it showed a water vapor transmission rate (WVTR) of $7.87 \times 10^{-6} \mathrm{~g} \mathrm{~m}^{-2} \mathrm{day}^{-1}$. It retained a WVTR value of $7.78 \times 10^{-5} \mathrm{~g} \mathrm{~m}^{-2}$ day $^{-1}$ under strain of nearly $0.63 \%$. A $3 \mu \mathrm{m}$-thick thin PET film was used as the liquid passivation, and it protected the textile-OLED from physical damage, and functioned as the blocking layer against rain, sweat, and other water encountered in daily life. As shown in Fig. $4 \mathrm{f}$ and $\mathrm{g}$, the encapsulated textile-OLEDs kept more than $90 \%$ of their luminance during $200 \mathrm{~min}$ under water. The water probably permeated into the gaps between the textile platform and the liquid passivation, rather than penetrating the gas barrier and the liquid passivation. 
a
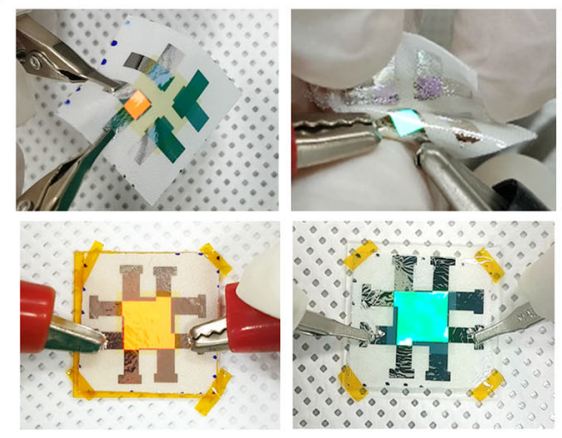

C

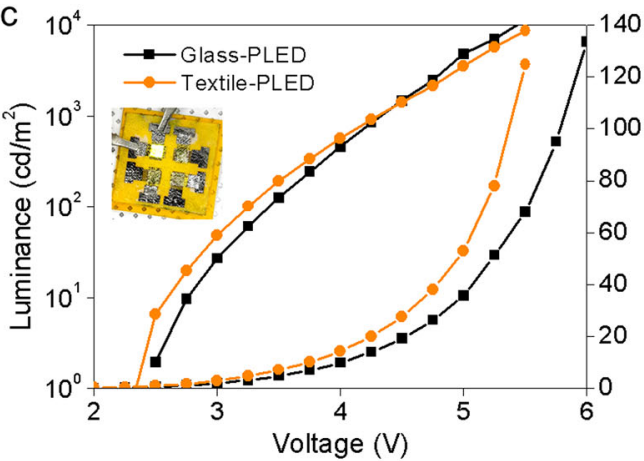

e

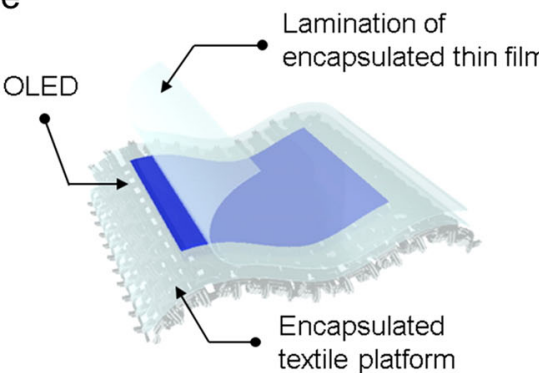

f

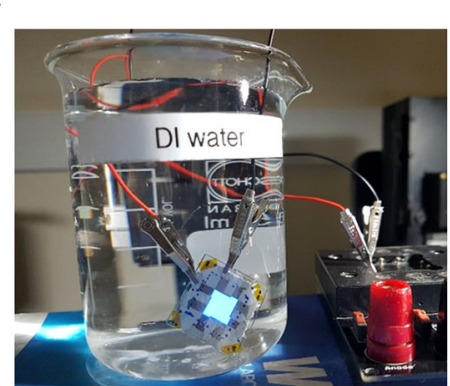

b

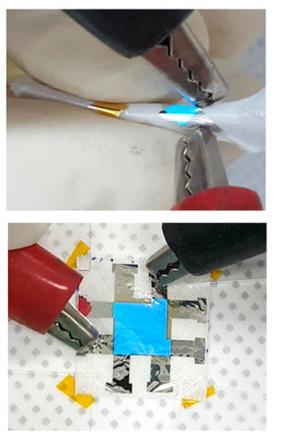

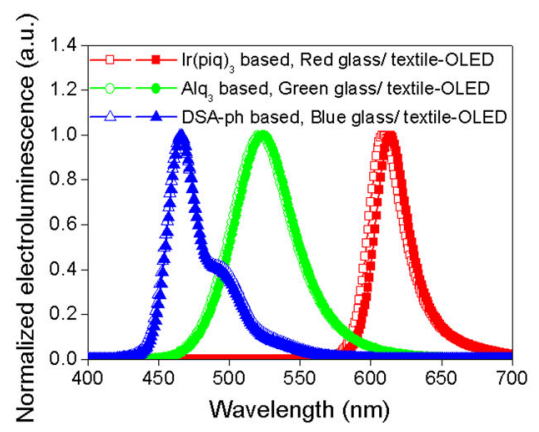

d

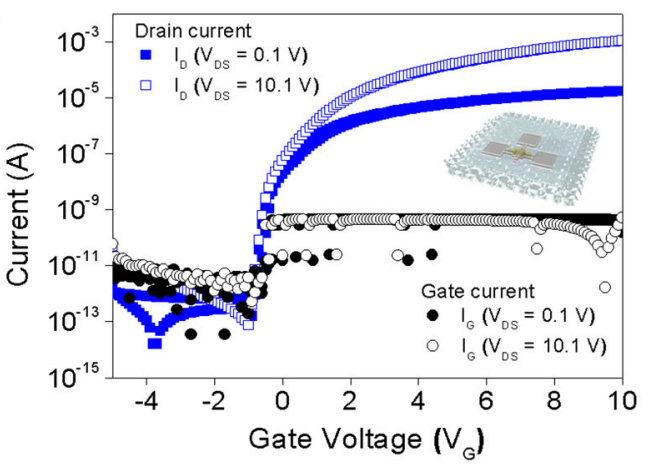

$\mathrm{g}$

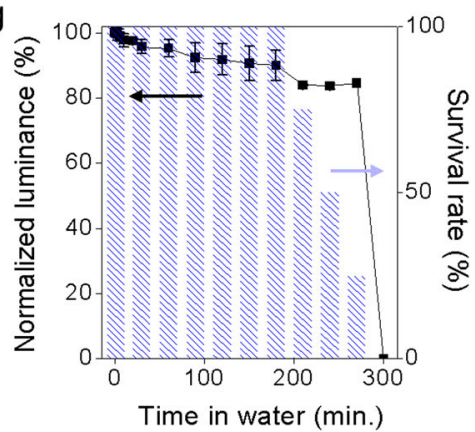

Fig. 4 Element technologies for textile-AMOLED, and wide applicability to various textiles. a The R, G, B colored textile-OLEDs under deformation, and with a wide area of $69 \mathrm{~mm}^{2}$. b Normalized electroluminescence of the R, G, B colored glass-OLED and textile-OLED. c Electrical and optical characteristics (JVL) of the glass-PLED and the textile-PLED. $\mathbf{d}$ Transfer curve of the textile-TFT (linear mode: $V_{\mathrm{DS}}=0.1 \mathrm{~V}$, saturation mode: $V_{D S}=10.1 \mathrm{~V}$ ). e Schematic diagram of the washable textile-OLED with the water-resistant encapsulation. $\mathbf{f}$ Photograph of the washable textile-OLED operating in DI water. $\mathbf{g}$ Changes in luminance over time (the initial luminance: 1000 nit).

Finally, all kinds of heat-sensitive textiles can be employed as the textile platform proposed here. The textile within the platform experienced no heating processes, because the strain buffer was hardened by reaction with moisture in the air at room temperature. We used a cotton, a linen, a wool, and a leather as well as polyester textiles to fabricate the stress-lowering structured textile platform, as shown in Fig. 5a and b. All of the textiles were conventional materials, and had not been processed for any characteristic enhancement. All the textiles were purchased from an ordinary fabric market.

Figure $5 \mathrm{c}$ shows a demonstration of the textile-OLED display in this work. The clothes with the wearable displays can provide information, such as today's weather, the time, schedules, the body conditions of a wearer, and news via connection with smart devices. The textile-display was also realized as a soft and comfortable watch on the shirtsleeve, as shown in Fig. $5 \mathrm{~d}$. A person, who has left his or her watch behind at home, or does not like wearing a watch, can have this smart watch by just wearing it. These textile-displays can highlight a logo on a cloth, and function as smart healthcare wear, and be premium clothing.

\section{DISCUSSION}

In summary, we demonstrated the essential elements and technologies of the highly flexible and wrinkle-able textile display. The flat surface of a glass substrate was perfectly replicated on the textile using just a $0.5 \mu \mathrm{m}$-thick planarization layer. The planarization layer was selected as the SU-8 considering a surface energy, high flexibility by ultra-thinness but no stretchability for low strain. The strain buffer reduced the large strains in the textile, and transferred a reduced strain to the device on the planarization layer. As confirmed from the cantilever test, the textile platform had flexibility like a real textile. We utilized the various tests, such as the peel test, tensile test, bending test, wrinkling test, DIC, etc., to investigate the mechanical characteristics of the proposed textile platform. The textile-OLEDs were demonstrated on various textiles such as polyester, cotton, linen, wool, and leather. In addition, we successfully realized PLEDs and TFTs on the generally used polyester textile for the first time by using the planarization layer with a high heat resistance and a low CTE. Furthermore, we tested the commercial potential of the textile-display by demonstrating washable encapsulation, and specific demonstrations of practical applications. We expect this work to be a 
a
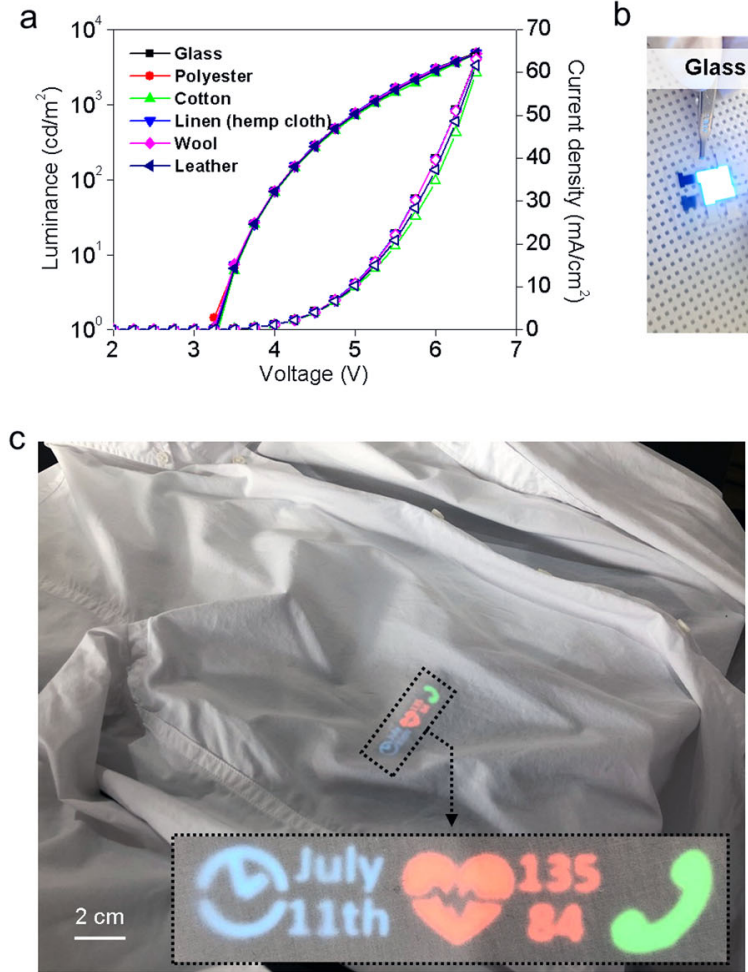

b

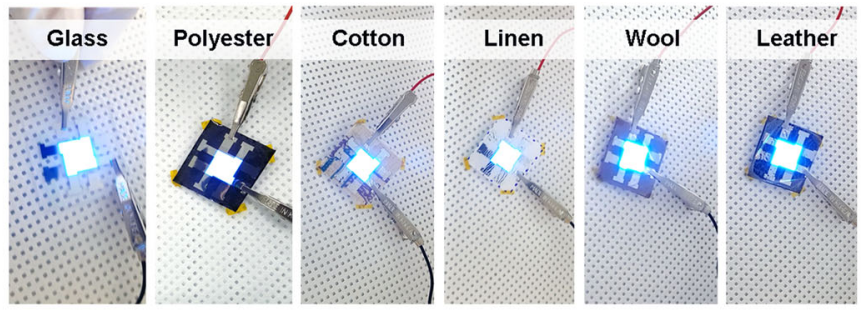

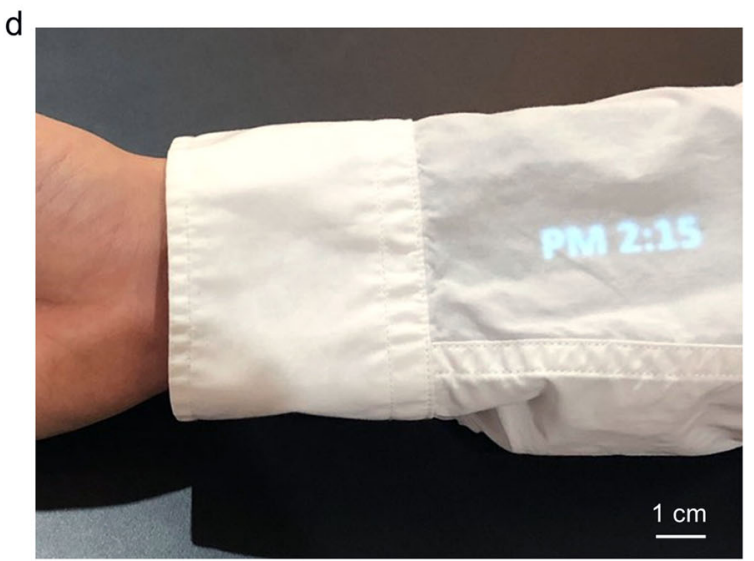

Fig. 5 Wide applicability of textile-OLED displays. a JVL characteristics of the textile-OLEDs fabricated with five different types of textiles (reference: OLED fabricated on a glass substrate). b Cell images of the textile-OLEDs applied to different types of textiles. c Display of a watch, the date, the heart rate, and phone call using textile-OLEDs. d Smart fashion displaying the current time on a shirt sleeve using the textileOLEDs.

springboard for the growth of textile-based wearable electronics, and to be helpful research for the development of e-textile industries.

\section{METHODS}

Fabrication of the textile platform

The sacrificial layer, the planarization layer, and the strain buffer were formed on the glass, and the textile was laminated. After the sacrificial layer was removed, the textile platform was obtained. A glass square $8.5 \mathrm{~cm} \times 8.5 \mathrm{~cm}$ was used to uniformly coat the high-viscosity materials. The glass was rinsed with DI water. The sacrificial layer, PVA, was filtered using a cellulose acetate filter with a pore size of $0.45 \mu \mathrm{m}$. The $1 \mu \mathrm{m}$-thick PVA layer was formed by spin-coating, and it was dried at $130^{\circ} \mathrm{C}$ for $10 \mathrm{~min}$ on a hotplate. The PVA solution was made by melting PVA powder in DI water at $90^{\circ} \mathrm{C}$ for more than $24 \mathrm{~h}$. The $0.5 \mu \mathrm{m}$ planarization layer, SU-8, was formed by spin-coating, soft-baked at $130^{\circ} \mathrm{C}$ for $1 \mathrm{~min}$, hardened by UV exposure of $56 \mathrm{~mJ}$ and post exposure baked (PEB) at $130^{\circ} \mathrm{C}$ for $1 \mathrm{~min}$. After the 7-10 $\mu \mathrm{m}$-thick strain buffer was formed by the spin-coating, the textile was laminated. Before the hardening process of the strain buffer, the sample was uniformly pressed with a force of $10 \mathrm{~N}$. The strain buffer was hardened at the room temperature, and it takes more than $2 \mathrm{~h}$. The PVA layer was easily removed by $\mathrm{DI}$ water, and the textile platform was separated from the glass. The textile platform was naturally dried for more than $24 \mathrm{~h}$.

Internal stress measurement

The internal stress was calculated by changes in the curvature of a 6-inch $\mathrm{Si}$ wafer using 500TC of Frontier Semiconductor Inc. After the initial curvature of the Si wafer was measured, the SU-8 was formed on the wafer. Because the internal stress of the SU- 8 caused a fine variation in the curvature of the Si wafer, the internal stress of the SU-8 could be calculated by measuring the change in the curvature of the $\mathrm{Si}$ wafer. The effect of the strain buffer and the textile on the internal stress were also determined using the same process.
Peel test

The peel tester consisted of a stage 1, holding the substrate, and a stage 2 holding the film to be peeled off the substrate. The SU-8 and the strain buffer were formed on the glass with a width of $1 \mathrm{~cm}$ and a length of $4 \mathrm{~cm}$, and the textile had a width of $1 \mathrm{~cm}$ and a length of $6 \mathrm{~cm}$. The glass was fixed to stage 1 , and the longer portion of the textile was held to stage 2 . Stages 1 and 2 were positioned to be perpendicular. Stage 2 moved in the direction for delamination, and stage 1 moved to maintain the delamination angles of $90^{\circ}$. The peeling force was measured during the test in stage 2 .

\section{Cantilever test}

The cantilever test is a method for measuring the flexibility of textiles. The cantilever tests in this work were conducted according to the international standard, American Society for Testing and Materials (ASTM D:1388). (The measurement method is mentioned in the main content and Fig. 1e.) Typically, cantilever tests are conducted in various directions because the woven structure affects the cantilever test results. However, there was not much difference in different directions for the textile used here, thus this was not indicated in the paper. The weights of the $6.25 \mathrm{~cm}^{2}$ samples were: the bare polyester textile $0.0695 \mathrm{~g}$; the textile platform $0.0776 \mathrm{~g}$; PET $50 \mu \mathrm{m}$ $0.0471 \mathrm{~g}$; PET $100 \mu \mathrm{m} 0.0953 \mathrm{~g}$; and A4 paper $0.0530 \mathrm{~g}$. These values were used to calculate the maximum bending moment, the bending rigidity and Young's modulus.

\section{Tensile test}

Tensile tests were conducted for the textile platform and each layer. The strain and the stress were calculated using the distance between two supports and the applied force needed to stretch the samples. Young's modulus of each sample was calculated in the linear elastic region, where the samples maintained mechanical properties without permanent deformation. 


\section{Mechanical simulation}

The ANSYS mechanical, structural mechanics calculation tool using FEM, was used to simulate bending strain of the textile platform. Based on the applied mechanics, the textile was modeled as a plate in the cantilever test analysis. Because Young's modulus values by the cantilever tests were inputted in the ANSYS simulation, plate modeling of the textile was also utilized in the simulation. One edge of the plate was a fixed support and the other edge was a displacement support. When the displacement support moved toward the center, bending of the plate was induced in the simulation. The Poisson's ratios of the SU-8, the strain buffer and the textile were inputted as $0.22,0.49$, and 0.40 , respectively ${ }^{39}$.

\section{OLED fabrication}

OLEDs with top emission and an inverted structure were fabricated on the glass and the textile platforms. All metals and organic layers were formed by thermal evaporation. The red-colored OLEDs consisted of $\mathrm{N}_{1} \mathrm{~N}^{\prime}-\mathrm{Bis}$ (naphthalen-1-yl)-N,N'-bis(phenyl)benzidine (NPB) $(50 \mathrm{~nm}), \mathrm{Ag}(30 \mathrm{~nm})$, $\mathrm{MoO}_{3}(5 \mathrm{~nm}), \mathrm{NPB}(65 \mathrm{~nm})$, Bis(10-hydroxybenzo[h]quinolinato)beryllium (Bebq2) doped with Tris[1-phenylisoquinolinato-C2,N]iridium(III) (Ir(piq)3) of $8 \mathrm{vr} \%(70 \mathrm{~nm})$, lithium quinolate (Liq) $(1 \mathrm{~nm})$ and $\mathrm{Al}(100 \mathrm{~nm})$. For the green-colored OLEDs, NPB $(50 \mathrm{~nm}), \mathrm{Ag}(30 \mathrm{~nm}), \mathrm{MoO}_{3}(5 \mathrm{~nm}), \mathrm{NPB}(50 \mathrm{~nm})$, Tris(8-hydroxyquinolinato)aluminum ( $\left.\mathrm{Alq}_{3}\right)(50 \mathrm{~nm})$, Liq $(1 \mathrm{~nm}), \mathrm{Al}(100 \mathrm{~nm})$ were used. For the blue-colored OLEDs, NPB $(50 \mathrm{~nm}), \mathrm{Ag}(30 \mathrm{~nm}), \mathrm{MoO}_{3}$ $(5 \mathrm{~nm}), \quad \mathrm{NPB} \quad(45 \mathrm{~nm}), \quad 2-$-Methyl-9,10-bis(naphthalen-2-yl)anthracene (MADN) doped with $\mathrm{p}$-di(p-N,N-diphenyl-amino-styryl)benzene (DSA-Ph) of $3 \mathrm{vr} \%(25 \mathrm{~nm}), \mathrm{Alq}_{3}(10 \mathrm{~nm})$, Liq $(1 \mathrm{~nm})$, Al $(100 \mathrm{~nm})$ were used. The $\mathrm{Ag}$ and Al were deposited at a rate of $2 \AA / s, B^{2} b_{2}$ and MADN at $3 \AA / s, \operatorname{lr}(\text { piq) })_{3}$ at $0.24 \AA / \mathrm{s}, \mathrm{DSA}-\mathrm{Ph}$ at $0.09 \AA \AA \mathrm{s}$, and the other at $1 \AA / \mathrm{s}$.

\section{PLED fabrication}

The PLEDs were fabricated using a structure of $\mathrm{ZnS}(20 \mathrm{~nm}), \mathrm{Ag}(100 \mathrm{~nm})$, $\mathrm{ZnO}(20 \mathrm{~nm})$, polyethylenimine (PEI) (sub-10 nm), PPV-based copolymer PDY-132 (Superyellow, Merck) $(70 \mathrm{~nm}), \mathrm{MoO}_{3}(10 \mathrm{~nm}), \mathrm{Ag}(20 \mathrm{~nm}), \mathrm{NPB}$ $(50 \mathrm{~nm}) . \mathrm{ZnS}, \mathrm{Ag}$, and $\mathrm{MoO}_{3}$ were deposited by thermal evaporation at a rate of 1,2 , and $0.5 \AA / s$, respectively. ZnO, PEl, PDY-132, were spin-coated and dried on a hot plate at $120^{\circ} \mathrm{C}$ in an $\mathrm{N}_{2}$ environment glove box.

\section{IGZO-TFT fabrication}

TFTs were fabricated with a top-gate and staggered structure on the textile platform. Transparent and flexible three-layer electrodes of indium tin oxide (ITO)-Ag-ITO, called IAI was used as the gate, the source, and the drain. ITO and Ag were deposited by sputtering and thermal evaporation, respectively. Each layer of the IAI electrode was optimized as 51, 15, and $45 \mathrm{~nm}$ for high transparency. The sheet resistance of the IAI electrode was $6.55 \Omega$ /square, and luminous transmittance was $84.72 \%$. The IAI electrode maintained its initial sheet resistance after the 10,000th repeated bending at a bending strain of $0.6 \%$. The insulator, an $\mathrm{Al}_{2} \mathrm{O}_{3}$ layer of $40 \mathrm{~nm}$, was formed by ALD at $150^{\circ} \mathrm{C}$, and its dielectric constant was 8.36 at $100 \mathrm{~Hz}$. Amorphous indium gallium zinc oxide (a-IGZO) of $20 \mathrm{~nm}$ was used as the active layer, and deposited by sputtering. In the TFT fabrication process, there was no photolithography process, but just vacuum deposition by shadow masks, in order to avoid deformation of the textile by solutions or heating process.

\section{Nano-stratified and washable encapsulation}

The ALD system, which can produce dense films, was used to form the nano-stratified barrier. The nano-stratified barrier consisted of five pairs of $\mathrm{Al}_{2} \mathrm{O}_{3}$ and $\mathrm{ZnO}$ quasi-films. The thickness of the $\mathrm{Al}_{2} \mathrm{O}_{3}$ and $\mathrm{ZnO}$ layers was $3 \mathrm{~nm}$, thus the total thickness of the barrier was $30 \mathrm{~nm}$. The WVTR of the nano-stratified barrier with the multi-interface system was lower than that of the single $\mathrm{Al}_{2} \mathrm{O}_{3}$ and $\mathrm{ZnO}$ layers of $30 \mathrm{~nm}$, because the nano-stratified barrier had longer diffusion path lengths. In addition, trimethyaluminum (TMA), the precursor of $\mathrm{Al}_{2} \mathrm{O}_{3}$, was used to etch the $\mathrm{ZnO}$ layer, which resulted in micro-cracks inside the barrier. The micro-cracks interrupted the growth of serious cracks produced by external forces, and thus the durability of the barrier under high strain was enhanced. The OLED encapsulation was fabricated by alternative depositions, 3.5 dyads, of the nano-stratified barrier and the polymer film, supported by Intech.

\section{DATA AVAILABILITY}

The data that support the findings of this study (and displayed in the figures) are available from the corresponding author upon reasonable request.

Received: 12 November 2019; Accepted: 28 October 2020; Published online: 26 November 2020

\section{REFERENCES}

1. Kwon, S. et al. Recent progress of fiber shaped lighting devices for smart display applications-fibertronic perspective. Adv. Mater. 32, 1903488 (2020).

2. Lee, S. M., Kwon, J. H., Kwon, S. \& Choi, K. C. A review of flexible OLEDs toward highly durable unusual displays. IEEE Trans. Electron Devices 64, 1922-1931 (2017).

3. O'Connor, B., An, K. H., Zhao, Y., Pipe, K. P. \& Shtein, M. Fiber shaped organic light emitting device. Adv. Mater. 19, 3897-3900 (2007).

4. Kwon, S. et al. Weavable and highly efficient organic light-emitting fibers for wearable electronics: a scalable, low-temperature process. Nano Lett. 18, 347-356 (2018).

5. Kwon, S. et al. High luminance fiber-based polymer light-emitting devices by a dip-coating method. Adv. Electron. Mater. 1, 1-8 (2015).

6. Rein, M. et al. Diode fibres for fabric-based optical communications. Nature 560, 214-218 (2018).

7. Zhang, Z. et al. Flexible electroluminescent fiber fabricated from coaxially wound carbon nanotube sheets. J. Mater. Chem. C 3, 5621-5624 (2015).

8. Zhang, Z. et al. Textile display for electronic and brain-interfaced communications. Adv. Mater. 30, 1-8 (2018).

9. Zhang, Z. et al. A colour-tunable, weavable fibre-shaped polymer light-emitting electrochemical cell. Nat. Photonics 9, 233-238 (2015).

10. Liang, G. et al. Coaxial-structured weavable and wearable electroluminescent fibers. Adv. Electron. Mater. 3, 1-10 (2017).

11. Cochrane, C., Meunier, L., Kell, F. M. \& Koncar, V. Flexible displays for smart clothing: Part I-overview. Indian J. Fibre Text. Res. 36, 429-435 (2011).

12. Meunier, L., Kell, F. M., Cochrane, C. \& Koncar, V. Flexible displays for smart clothing: Part I-overview. Indian J. Fibre Text. Res. 36, 422-428 (2011).

13. Cherenack, K. \& Van Pieterson, L. Smart textiles: challenges and opportunities. J. Appl. Phys. 112, 091301 (2012).

14. Hu, B. et al. Textile-based flexible electroluminescent devices. Adv. Funct. Mater. 21, 305-311 (2011).

15. Kim, W. et al. Soft fabric-based flexible organic light-emitting diodes. Org. Electron. Phys. Mater. Appl. 14, 3007-3013 (2013).

16. Kim, W. et al. Reliable actual fabric-based organic light-emitting diodes: toward a wearable display. Adv. Electron. Mater. 2, 1-7 (2016).

17. Choi, S. et al. Highly flexible and efficient fabric-based organic light-emitting devices for clothing-shaped wearable displays. Sci. Rep. 7, 1-8 (2017).

18. Kim, H., Kwon, S., Choi, S. \& Choi, K. C. Solution-processed bottom-emitting polymer light-emitting diodes on a textile substrate towards a wearable display. J. Inf. Disp. 16, 179-184 (2015).

19. De Vos, M., Torah, R., Glanc-Gostkiewicz, M. \& Tudor, J. A complex multilayer screen-printed electroluminescent watch display on fabric. J. Disp. Technol. 12, 1757-1763 (2016).

20. Jeong, E. G., Jeon, Y., Cho, S. H. \& Choi, K. C. Textile-based washable polymer solar cells for optoelectronic modules: toward self-powered smart clothing. Energy Environ. Sci. 12, 1878-1889 (2019).

21. Cho, S. H. et al. Plasmonically engineered textile polymer solar cells for highperformance, wearable photovoltaics. ACS Appl. Mater. Interfaces 11, 20864-20872 (2019).

22. Plaut, R. H. Formulas to determine fabric bending rigidity from simple tests. Text. Res. J. 85, 884-894 (2015).

23. Ghosh, T. K. \& Zhou, N. Characterization of fabric bending behavior: a review of measurement principles. Indian J. Fibre Text. Res. 28, 471-476 (2003).

24. de Bilbao, E., Soulat, D., Hivet, G. \& Gasser, A. Experimental study of bending behaviour of reinforcements. Exp. Mech. 50, 333-351 (2010).

25. Szablewski, P. \& Kobza, W. Numerical analysis of Peirce's cantilever test for the bending rigidity of textiles. Fibres Text. East. Eur. 11, 54-57 (2003).

26. Jeon, Y. et al. A wearable photobiomodulation patch using a flexible redwavelength OLED and its in vitro differential cell proliferation effects. Adv. Mater. Technol. 3, 1-10 (2018).

27. Lee, M. T., Liao, C. H., Tsai, C. H. \& Chen, C. H. Highly efficient, deep-blue doped organic light-emitting devices. Adv. Mater. 17, 2493-2497 (2005).

28. Lee, M. T., Chen, H. H., Liao, C. H., Tsai, C. H. \& Chen, C. H. Stable styrylaminedoped blue organic electroluminescent device based on 2-methyl-9, 10-d/(2naphthyl)anthracene. Appl. Phys. Lett. 85, 3301-3303 (2004). 
29. Jeon, S. O., Jeon, Y. M., Kim, J. W., Lee, C. W. \& Gong, M. S. A blue organic emitting diode derived from new styrylamine type dopant materials. Synth. Met. 157, 558-563 (2007).

30. $\mathrm{Yu}, \mathrm{J}$. et al. Enhancement of efficiency and lifetime of blue organic light-emitting diodes using two dopants in single emitting layer. Adv. Mater. Sci. Eng. 2012, 247976 (2012).

31. So, F. \& Kondakov, D. Degradation mechanisms in small-molecule and polymer organic light-emitting diodes. Adv. Mater. 22, 3762-3777 (2010).

32. Burrows, P. E. et al. Ultra barrier flexible substrates for flat panel displays. Displays 22, 65-69 (2001).

33. Graff, G. L., Williford, R. E. \& Burrows, P. E. Mechanisms of vapor permeation through multilayer barrier films: lag time versus equilibrium permeation. J. Appl. Phys. 96, 1840-1849 (2004).

34. Jeong, E. G., Han, Y. C., Im, H. G., Bae, B. S. \& Choi, K. C. Highly reliable hybrid nanostratified moisture barrier for encapsulating flexible OLEDs. Org. Electron. Phys. Mater. Appl. 33, 150-155 (2016).

35. Jeong, E. G. et al. A mechanically enhanced hybrid nano-stratified barrier with a defect suppression mechanism for highly reliable flexible OLEDs. Nanoscale 9, 6370-6379 (2017).

36. Kwon, J. H. et al. Design of highly water resistant, impermeable, and flexible thinfilm encapsulation based on inorganic/organic hybrid layers. ACS Appl. Mater. Interfaces 11, 3251-3261 (2019).

37. Kwon, J. H., Jeon, Y. \& Choi, K. C. Robust transparent and conductive gas diffusion multibarrier based on $\mathrm{Mg}$ - and $\mathrm{Al}$-doped $\mathrm{ZnO}$ as indium tin oxide-free electrodes for organic electronics. ACS Appl. Mater. Interfaces 10, 32387-32396 (2018).

38. Kwon, J. H. et al. Functional design of highly robust and flexible thin-film encapsulation composed of quasi-perfect sublayers for transparent, flexible displays. ACS Appl. Mater. Interfaces 9, 43983-43992 (2017).

39. Jang, K. I. et al. Rugged and breathable forms of stretchable electronics with adherent composite substrates for transcutaneous monitoring. Nat. Commun. $\mathbf{5}$ 1-10 (2014).

\section{ACKNOWLEDGEMENTS}

This work was supported by the National Research Foundation of Korea (NRF) grant funded by the Korea government (MSIT) (No. 2019R1A2C3010012), the Engineering Research Center of Excellence (ERC) Program supported by National Research Foundation (NRF), Korean Ministry of Science \& ICT (MSIT) (Grant No. NRF2017R1A5A1014708), and the Technology Innovation Program (20000489, Interactive fiber-based wearable display platforms for clothing displays) funded by the Ministry of Trade, Industry \& Energy (MOTIE, Korea).

\section{AUTHOR CONTRIBUTIONS}

S.C. and K.C.C. conceived and designed the research. S.C. mainly conducted the experiments and analyses, and prepared figures and the manuscript. W.J. contributed to mechanical analyses, and conducted the peel test and the tensile test. S.K. designed the research object and specific experimental methods. Y.J. and J.H.K. optimized OLEDs and OLED encapsulation. Y.H.S. analyzed the mechanical bending characteristics of the textile platform. J.K. designed the experimental method of DIC and analyzed DIC results. J.H.P designed and fabricated the textile-TFTs. H.K. and H.S. L. designed and fabricated the solution-processed textile-PLEDs. M.N. contributed the design and the fabrication of the textile platform. E.G.J. developed the nano-stratified encapsulation. J.B.S. provided useful advice throughout the research. T.-S.K. contributed mechanical characteristics evaluation of this work.

\section{COMPETING INTERESTS}

The authors declare no competing interests.

\section{ADDITIONAL INFORMATION}

Supplementary information is available for this paper at https://doi.org/10.1038/ s41528-020-00096-3.

Correspondence and requests for materials should be addressed to K.C.C.

Reprints and permission information is available at http://www.nature.com/ reprints

Publisher's note Springer Nature remains neutral with regard to jurisdictional claims in published maps and institutional affiliations.

(i) Open Access This article is licensed under a Creative Commons Attribution 4.0 International License, which permits use, sharing, adaptation, distribution and reproduction in any medium or format, as long as you give appropriate credit to the original author(s) and the source, provide a link to the Creative Commons license, and indicate if changes were made. The images or other third party material in this article are included in the article's Creative Commons license, unless indicated otherwise in a credit line to the material. If material is not included in the article's Creative Commons license and your intended use is not permitted by statutory regulation or exceeds the permitted use, you will need to obtain permission directly from the copyright holder. To view a copy of this license, visit http://creativecommons. org/licenses/by/4.0/.

(c) The Author(s) 2020 